\title{
3D-Objekterkennung mit Jetson Nano und Integration mit KUKA KR6-Roboter für autonomes Pick-and-Place
}

\author{
Akhila Pullela ${ }^{1}$, Elmar Wings ${ }^{2}$
}

\section{Zusammenfassung}

Bildverarbeitungssysteme bieten innovative Lösungen für den Fertigungsprozess. Kameras und zugehörige Bildverarbeitungssysteme können zur Identifizierung, Prüfung und Lokalisierung von Teilen auf einem Förderband oder in einem Behälter mit Teilen eingesetzt werden. Roboter werden dann eingesetzt, um jedes Teil aufzunehmen und im Montagebereich zu platzieren oder sogar um die Grundmontage direkt durchzuführen. Das System für dieses Projekt besteht aus einem Roboter Kuka KR6 900, der die Position (x-, y- und z-Koordinaten des Objektschwerpunkts) und die Ausrichtung eines Bauteils von einem Bildverarbeitungssystem basierend auf einem Jetson Nano erhält. Das Ziel dieses Projekts ist es, eine automatische Erkennung eines Objekts mit Hilfe einer 2D-Kamera und der Auswertung mit dem Deep Learning Algorithmus Darknet YOLO V4 [1] durchzuführen, so dass der Roboter das Objekt greifen und platzieren kann. Dieses Projekt verwendet zwei verschiedene Objekttypen: einen Quader und einen Zylinder. Die Bilderkennung erfolgt mit Hilfe des Jetson Nano, dort erfolgt aus den Pixelkoordinaten die Berechnung der realen Koordinaten, die dann über die TCP/IP-Schnittstelle des Kuka KR6 900 zur Durchführung der Entnahme und Platzierung übermittelt werden. Die Flexibilität des Roboters, dessen Steuerung auf diese Weise von der Bildverarbeitung unterstützt wird, kann den Bedarf an präzise konstruierten Teilezuführungen verringern und so die Flexibilität in der Fertigungszelle erhöhen und kurze Produktionsläufe und Anpassungsfähigkeit ermöglichen.

\section{Stichwörter}

Jetson Nano, Kuka, Bilderkennung, yolo, Pick-and-Place

\section{Stand der Technik}

Pei-Chi Huang und Aloysius K. Mok [2] schlugen 2018 eine Methode für die Amazon Picking Challenge vor, bei der die Informationen vom Xtion-Kamerasensor für etwa 18 Objekte geliefert wurden und ein untexturiertes 3D-Netz durch Object Recognition Kitchen rekonstruiert wurde. Anschließend wurden alle Objektnetze und idealen Griffpositionen in einer CouchDB-Datenbank für spätere Abfragen gespeichert. Um das Zielobjekt in einer Gruppe von Objekten zu erkennen, wurde YOLO eingesetzt, um einen Deep-Learning-Klassifikator zu trainieren, der die Objekte erkennt und die gewünschte Region of Interest (ROI) in einer Kameraansicht in Echtzeit liefert. LineMOD wurde für den Vorlagenabgleich verwendet, um die Position des Objekts zu ermitteln.

Shete, Pritam Prakash und andere [3] schlugen 2015 ein stereobildgesteuertes Telerobotiksystem für autonome Pick-And-Place-Operationen vor. Sie verwendeten ein Paar IP-Kameras als Stereokamera, einen 3D-fähigen Monitor für die Anzeige von Stereoinhalten und ein NVIDIA 3D Vision Pro System für die Verwaltung. Die Koordinatensysteme des Roboters und des stereoskopischen Systems werden

$1 \quad$ B.Eng. Akhila Pullela, HS Emden-Leer, D-26723 Emden

2 Prof. Dr. rer.nat Elmar Wings, HS Emden-Leer, D-26723 Emden 
mithilfe eines planaren Schachbrett-Kalibrierungsmusters in ein vordefiniertes Weltkoordinatensystem transformiert. Für das stereoskopische System wird ein Fehler von weniger als $2 \mathrm{~mm}$ in X- und Y-Richtung und weniger als $5 \mathrm{~mm}$ in Z-Richtung, der Blickrichtung, angegeben.

T. Kotthauser und G. F. Mauer [4] schlugen eine Methode vor, bei der die Kamerakalibrierung anhand von Kalibrierungsobjekten mit bekannter Geometrie durchgeführt wird, die an bekannten räumlichen Positionen von der Kamera platziert werden. Die räumlichen Positionen werden mittels Triangulation in Matlab rekonstruiert. Eine 3D-Szene wurde aus 2 ebenen Bildern unter Verwendung der epipolaren Geometrie rekonstruiert. Die Fundamentalmatrix, die beliebige Punkte in der 3D-Szene mit ihrem jeweiligen planaren Bild verbindet, wurde mit dem Acht-Punkte-Algorithmus gefunden.

P. Andhare und S. Rawat [5] schlugen eine Methode vor, bei der sie einen Industrieroboter so programmierten, dass er mit Hilfe des maschinellen Sehens ein Pick-And-Place-Verfahren durchführt. Die maschinelle Programmierung umfasst die Objekterkennung, die Bildvorverarbeitung, die Umwandlung von Pixelkoordinaten in Weltkoordinaten unter Verwendung von Transformationseigenschaften und die Berechnung der Ausrichtung des Objekts.

\section{Ziele des Projekts}

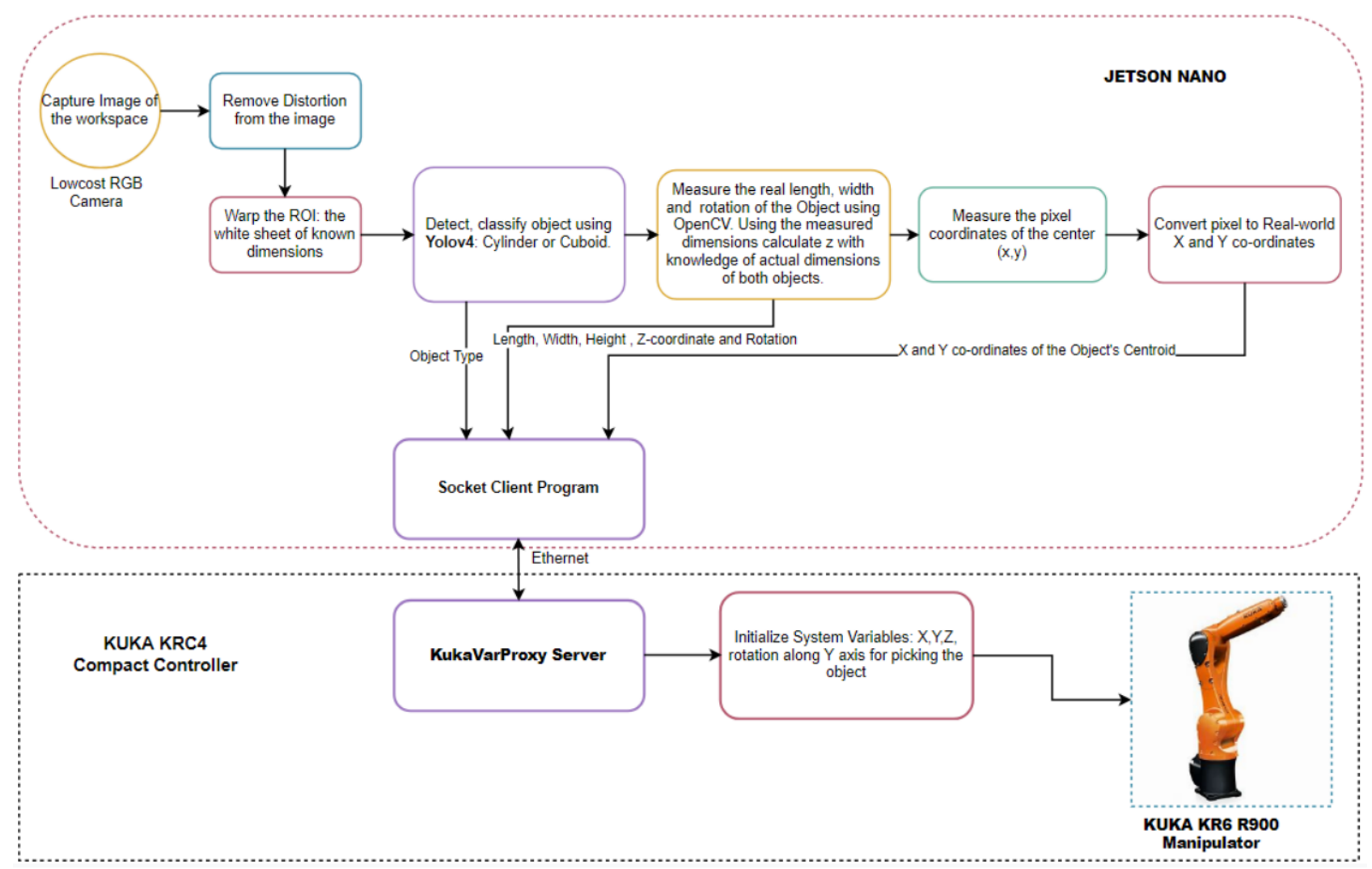

Bild 1: Blockdiagramm der Implementierung

Das grundsätzliche Problem dieser Arbeit ist die Auswahl und Platzierung von Objekten, die sich unter dem Vision-Sensor befinden. Der Sensor ist hier eine Logitech-Webkamera, die mit dem NVIDIA Jetson Nano verbunden ist. Dieser erfasst das Bild der Objekte, die auf einem definierten Arbeitsbereich platziert werden, erkennt und klassifiziert das Objekt mit Hilfe des Deep-Learning-Algorithmus YOLO V4, entzerrt und extrahiert den definierten Roboterarbeitsbereich aus dem erfassten Bild mit Hilfe von Konturerkennungsmethoden von OpenCV. Später wird das Objekt auf dem verzerrten Bild des Arbeitsbereichs mit der Canny-Edge-Detection-Methode von OpenCV lokalisiert und die realen Koordinaten des Schwerpunkts werden aus den Pixelkoordinaten mit Hilfe von Bildmomenten von OpenCV berech- 
net. Auch die reale Höhe, die reale Breite des Objekts und die Drehung, wie sie in einem zweidimensionalen Bild beobachtet werden, können mit Hilfe von Methoden aus OpenCV und NumPy aus den Pixeln gemessen werden. Diese Werte werden vom Jetson Nano über eine Client-Server-Kommunikation an die Kuka KRC4-Steuerung gesendet, und der Roboter wird so programmiert, dass er den Greifer an den gewünschten Ort bewegt. Der Referenzrahmen, d.h. eine Ecke des weißen Arbeitsbereiches auf dem Tisch als Ursprung, wird für den Roboter mit dem Programmierhandgerät definiert. Die Kameramatrix und die Verzerrungskoeffizienten der Kamera werden mit der Schachbrett-Kalibrierungsmethode berechnet und die Bilder werden zur Verbesserung der Genauigkeit entzerrt.

\section{Herausforderungen}

Die Objektklassifizierung erfolgt mit YOLO V4, das bei guter Beleuchtung besser abschneidet, wenn der Hintergrund des Objekts einfarbig und hell gefärbt ist. Das Training des Algorithmus für die beiden Objektklassen Zylinder und Quader dauerte in Google Colab aufgrund der Laufzeitbeschränkungen in Colab etwa 35 Stunden. Die Kamera wird in dieser Anwendung als visueller Sensor verwendet. Wenn eine Kamera 3D-Objekte in der realen Welt betrachtet und sie in ein 2D-Bild umwandelt, ist die Umwandlung nicht perfekt. Manchmal sind die Bilder verzerrt: Kanten werden gebogen, abgerundet oder nach außen gestreckt. Dies ist auf die tangentiale und radiale Verzerrung zurückzuführen, die meist durch das Kameraobjektiv und die Position der Kamera verursacht wird. In unserem Anwendungsfall, in dem die Informationen aus dem Bild entscheidend sind, ist es notwendig, diese Verzerrungen zu korrigieren. Um dies zu ermöglichen, werden die Kameramatrix und die Verzerrungskoeffizienten mit Hilfe von Schachbrett-Kalibrierungsmethoden berechnet und die Bilder mit Undistort-Methoden aus OpenCV unverzerrt.

\section{Einrichtung des Arbeitsplatzes}

Das System wurde in einem vereinfachten Aufbau definiert, der eine repräsentative Betriebsumgebung enthält, d.h. einen Tisch mit einer dunklen Oberfläche, auf dem ein weißer Arbeitsbereich mit bekannten Abmessungen definiert ist, auf dem die Objekte zufällig platziert werden können. Dies ist für die Konturerfassung hilfreich, da die Ränder des weißen Arbeitsbereiches leicht zu detektieren sind und die Objekte darauf leicht zu lokalisieren sind. Es wurde eine Logitech HD C270-Webcam mit USB 2.0 verwendet, um die Bilder des Arbeitsplatzes oben aufzunehmen. Der USB-Anschluss der Webcam ist mit dem Jetson Nano verbunden, siehe Bild 3, der außerhalb des Arbeitsbereichs des Roboters platziert ist, um Schäden zu vermeiden. Auf die Benutzeroberfläche des Jetson Nano kann aus der Ferne oder über einen Monitor zugegriffen werden. Der Kuka KR6 Manipulator hat 6 Freiheitsgrade und kann eine Nutzlast von $6 \mathrm{~kg}$ handhaben. Außerdem beträgt die maximale Reichweite des Manipulators in horizontaler Richtung $901 \mathrm{~mm}$. Ein pneumatischer 2-Finger-Greifer ist als Endeffektor am Manipulator KR6 900 montiert. Die KUKA KRC4 compact ist die einheitliche Steuerung für die Bewegungen des Manipulators. Ihre offene Architektur ermöglicht die Steuerung von KUKA-Robotern und externen Achsen. Das Programmierhandgerät ist das Kuka Smart Pad zur Fernsteuerung des Roboters. Der Roboter und seine Steuerung sind in dem Bild 3 dargestellt.

\section{Umsetzung der vorgeschlagenen Lösung}

Die gesamte Arbeit gliedert sich in die Bereiche Bildverarbeitung, Objekterkennung und -lokalisierung, Koordinatentransformation, Positionsberechnung, Einrichtung einer Client-Server-Architektur zwischen der Kuka KRC4-Steuerung und dem Jetson Nano zur Übermittlung der Koordinaten an den Roboter, damit dieser die erkannten Objekte aufnehmen und platzieren kann. 

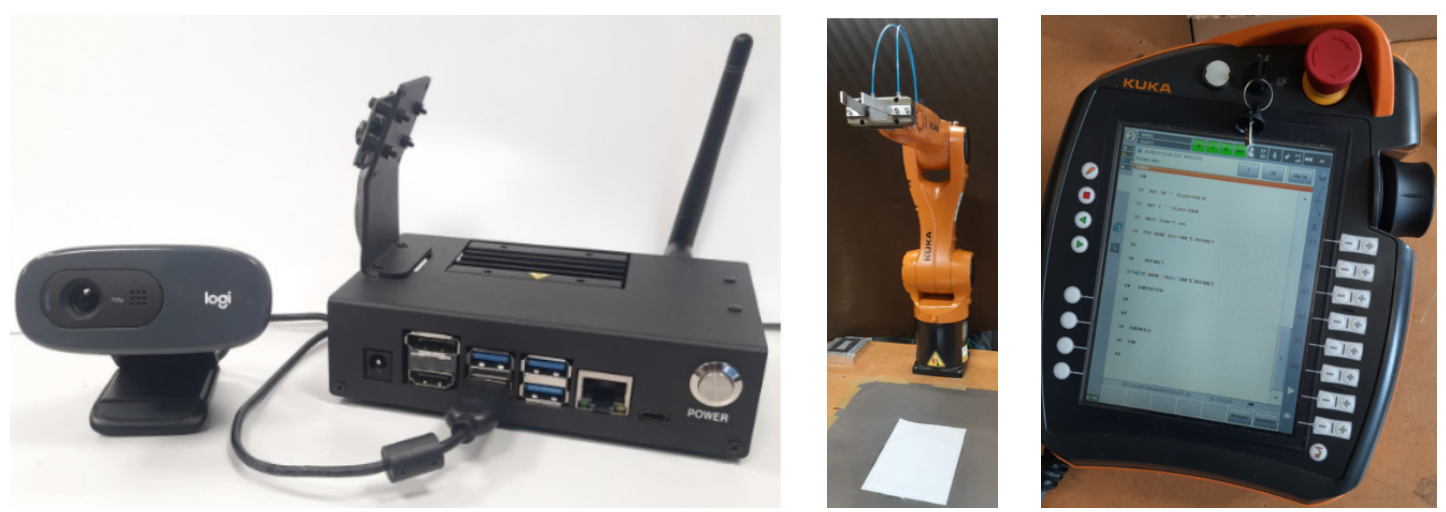

Bild 2: Jetson Nano mit Logitech-Webkamera, Kuka KR6 R900 und Smart Pad

\subsection{Objekterkennung mit dem Algorithmus YOLO V4}

Für die Objekterkennung wurde der Ansatz „Knowledge Discovery in Databases“ [6] verfolgt, der mehrere Schritte wie Datenauswahl, -bereinigung, -vorverarbeitung, -transformation, Datamining usw. umfasst. Es wurde eine Datenbank mit 672 Bildern für Quader und 696 Bildern für zylindrische Objekte in verschiedenen möglichen Ausrichtungen erstellt, indem kontinuierlich Bilder aus dem Livestream der Kamera des Jetson Nano aufgenommen wurden. Für die Vorbereitung des Datensatzes haben wir das YOLO-Beschriftungstool verwendet, mit dem wir Bounding Boxes gezeichnet und die Klassennummer als 0 für die zylindrischen und 1 für die quaderförmigen Objekte markiert haben. Mit diesem Tool wurde eine Textdatei erstellt, die die Klassennummer, die Objektkoordinaten, die Breite und die Höhe für jedes beschriftete Bild enthält. Wir haben ein vortrainiertes YOLO V4-Modell gewählt, das auf dem COCO-Datensatz eine durchschnittliche Genauigkeit von 43,5\% und eine Echtzeitgeschwindigkeit von 65 FPS auf einem Tesla V100 erreicht hat. Die Bildrate pro Sekunde ist der übliche Indikator für die Bewertung der Geschwindigkeit, d. h. die Anzahl der pro Sekunde verarbeiteten Bilder. Die durchschnittliche Präzision ist ein weiterer Parameter zur Messung der Genauigkeit eines Modells.

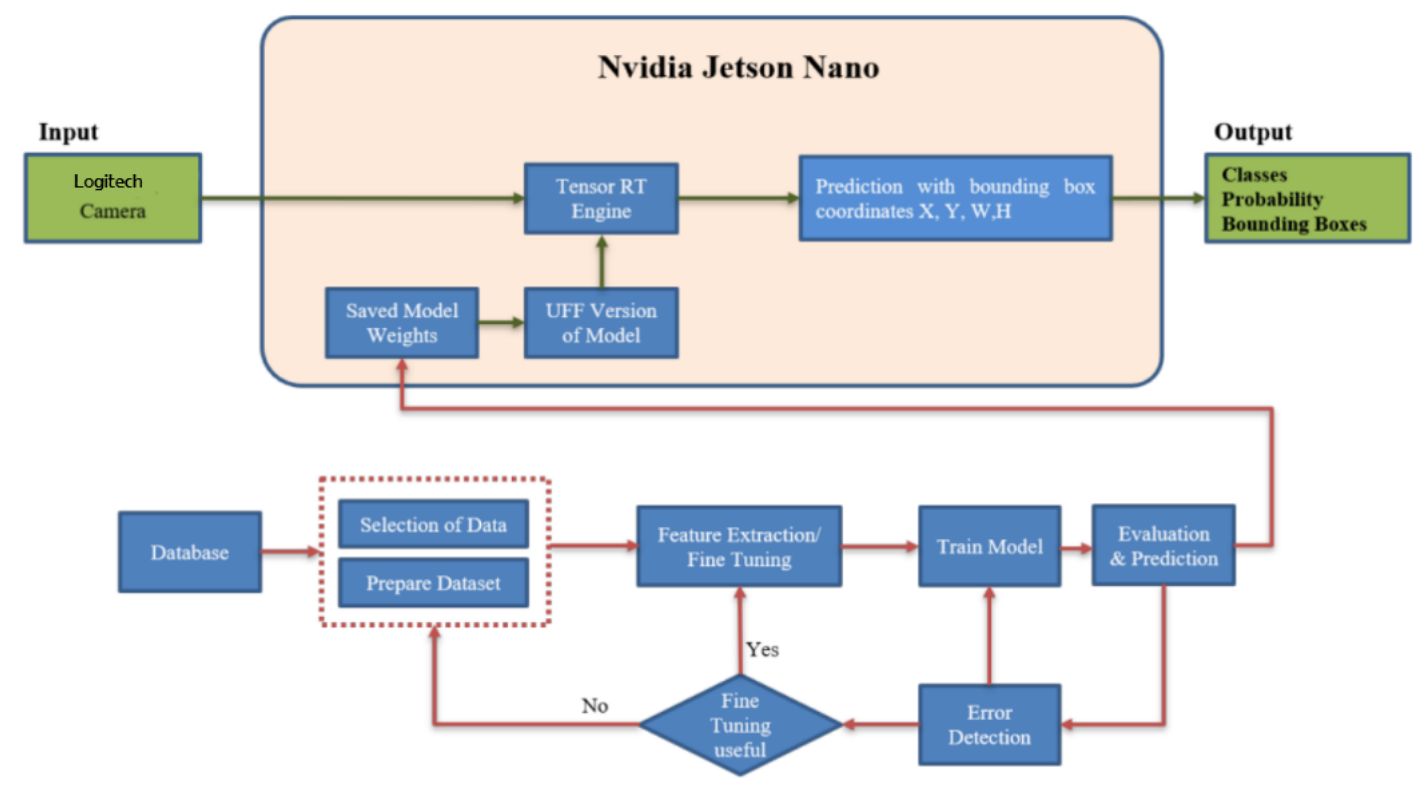

Bild 3: Eingesetzte Architektur für die Objekterkennung mit dem Jetson Nano

YOLO V4 besteht aus dem CSP Darknet53 als Backbone, einem tiefen neuronalen Netz, das hauptsächlich aus 53 Faltungsschichten besteht. Das Hauptziel des Backbones ist es, die wesentlichen Merkmale zu extrahieren. Zwischen dem Backbone und dem Head wird immer der Neck eingefügt, der Objekte in verschiedenen Maßstäben und räumlichen Auflösungen erkennen kann. Der Head 
eines Objektdetektors ist für die Klassifizierung und Lokalisierung zuständig. Spatial Pyramid Pooling (SPP) wendet maximales Pooling an, während Path Aggregation Network (PAN) angewendet wird, um Merkmale in einer hierarchischen Struktur abzubilden. Zum Trainieren des Modells wird das Darknet aus dem Darknet-Github-Repository in eine virtuelle Maschine geklont. Darknet ist ein OpenSource-Framework für neuronale Netze, das in C und CUDA geschrieben wurde und sowohl GPU als auch CPU unterstützt. Der Laufzeittyp und der Hardware-Beschleuniger sind in Colab Notebook auf GPU eingestellt. Die folgenden Änderungen werden an der Konfigurationsdatei von YOLO V4 vorgenommen:

- Batch Size $=64$ und Subdivisions $=16$

- Max Batches $=6000$, Steps $=4800,5400$

- Number of Filters $=21$

- $\quad$ Number of Classes= 2 (Zylinder and Quader) in den YOLO-Schichten in der Konfigurationsdatei.

- Learning rate $=.001$

- Breite und Höhe der Bilder $=416$

Ungefähr 1200 Bilder werden als Trainingsdatensatz ausgewählt und die restlichen Bilder werden als Validierungsdatensatz verwendet. Das Training dauerte aufgrund der Laufzeitbeschränkungen von Google Colab ca. 35 Stunden. Die Gewichte nach jeweils 100, 1000, 2000 Iterationen und die endgültigen Gewichte wurden gespeichert. Es wird festgestellt, dass der durchschnittliche Verlust des Modells für die letzten 1800 Iterationen unter 0,5 liegt und die durchschnittliche Genauigkeit 100\% beträgt.

Das Modell wird dann auf dem Jetson Nano eingesetzt. Der Jetson Nano hat eine Tegra-X1-System-onChip-Architektur. Es werden Änderungen an dem Makefile vorgenommen, um das Darknet für diese Architektur zu erstellen. Bei der Verwendung des Systems wird eine durchschnittliche Bildrate von 4,2 FPS erreicht. In der Abbildung 4 sind die beiden Objekte Beispielergebnisse dargestellt.
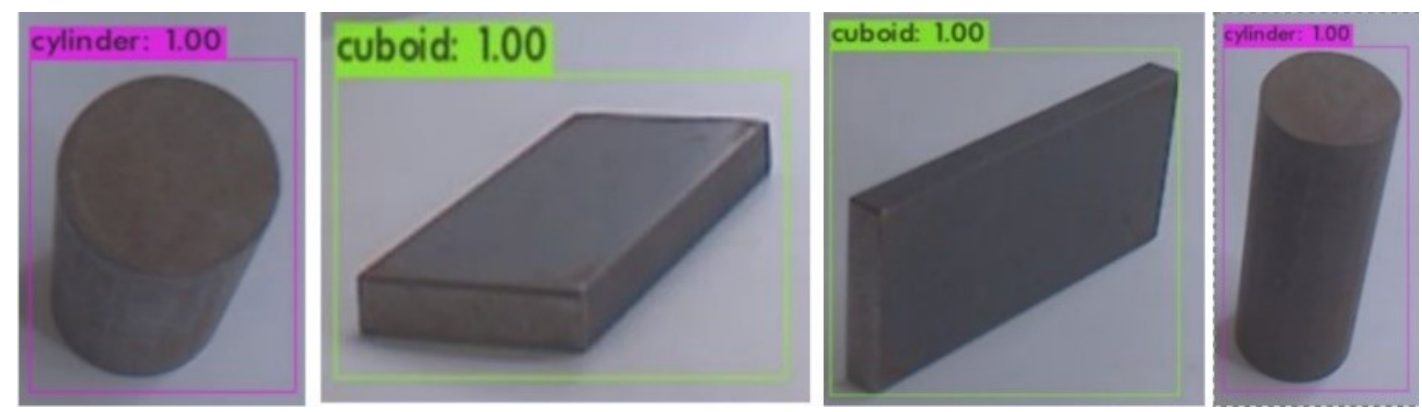

Bild 4: Beispiele von Vorhersagen des Modells zu den Objekten

\subsection{Kamerakalibrierung und Vermessung der Verzerrung}

Die Kamera wird in dieser Anwendung als visueller Sensor verwendet. Es ist wichtig, die Parameter der Kamera zu kennen, um sie effektiv als visuellen Sensor in unserer Anwendung zu nutzen. Die Kameraobjektive können zu Verzerrungen führen [7], da die Lichtstrahlen an den Rändern eines gekrümmten Kameraobjektivs oft ein wenig zu stark gebogen werden, was zu einer Verzerrung der Bildränder führt. Um die Verzerrungskoeffizienten und die Kameramatrix der Logitech-Webkamera zu messen, haben wir die Schachbrett-Kalibrierungsmethode [8] verwendet. Ein Schachbrett eignet sich hervorragend für die Kalibrierung, da es aufgrund seines regelmäßigen, kontrastreichen Musters leicht automatisch erkannt werden kann. Darüber hinaus sind die Ecken der Quadrate auf dem Schachbrett ideal für ihre Lokalisierung, da sie scharfe Farbverläufe in zwei Richtungen aufweisen. Darüber hinaus sind diese 
Ecken auch dadurch miteinander verbunden, dass sie sich am Schnittpunkt der Linien des Schachbretts befinden. All diese Fakten werden genutzt, um die Ecken der Quadrate in einem Schachbrettmuster zuverlässig zu lokalisieren.

Bei der Kalibrierung berechnen wir die Kameraparameter anhand einer Reihe bekannter 3D-Punkte (X, Y, Z) und ihrer entsprechenden Pixelposition (u, v) im Bild. Für die 3D-Punkte haben wir ca. 40 Bilder eines Schachbrettmusters mit bekannten Abmessungen in vielen verschiedenen Ausrichtungen aufgenommen. Die Weltkoordinaten sind mit dem Schachbrettmuster verbunden, und da alle Eckpunkte auf einer Ebene liegen, können wir Z für jeden Punkt willkürlich auf 0 setzen. Da die Punkte im Schachbrettmuster gleichmäßig verteilt sind, lassen sich die (X, Y)-Koordinaten jedes 3D-Punktes leicht definieren, indem man einen Punkt als Referenz $(0,0)$ nimmt und die übrigen in Bezug auf diesen Referenzpunkt definiert. Wir haben die OpenCV-Funktionen wie cv2.findchessboardcorners(), die die Ecken im Schachbrett aus dem Bild findet, und cv2.calibratecamera(), die die Bildpunkte und die Objektpunkte nimmt, verwendet. Die Koordinaten der Ecken im angezeigten 2D-Bild werden als Bildpunkte bezeichnet, die auf die 3D-Koordinaten der realen, unverzerrten Schachbrettecken, die als Objektpunkte bezeichnet werden, abgebildet werden. Die Z-Koordinaten bleiben unverändert, aber für die ersten beiden Spalten X und Y wird die Funktion mgrid von Numpy verwendet, um die gewünschten Koordinaten zu erzeugen. mgrid gibt die Koordinatenwerte für eine bestimmte Gittergröße zurück. Die Funktion cv2.calibratecamera() gibt die Kameramatrix und die Verzerrungskoeffizienten zurück. Diese Verzerrungskoeffizienten sind ein Maß für die radiale und tangentiale Verzerrung der Kamera. Die Bilder können mit der Funktion cv2.undistort() entzerrt werden, die die Verzerrungskoeffizienten und das verzerrte Originalbild aufnimmt und ein unverzerrtes Bild zurückgibt.

\subsection{Berechnung der Koordinaten der realen Welt aus den Pixelkoordinaten}

Da der Roboter Weltkoordinaten erwartet, werden die realen Koordinaten aus den Pixelkoordinaten des Jetson Nano ermittelt. Zunächst wurde eine Region von Interesse extrahiert, in der die Objekte lokalisiert werden sollen, die realen Abmessungen des Objekts wurden gemessen, der Schwerpunkt wurde berechnet, die realen Koordinaten in X- und Y-Achse des Objektschwerpunkts wurden gemessen, wobei eine Ecke des weißen Arbeitsbereiches als Ursprung verwendet wurde, und schließlich wurde die Drehung des Objekts in der 2D-Ebene gemessen. Für die Extraktion des interessierenden Bereichs wurde ein Programm geschrieben, das zunächst einen weißen Arbeitsbereich mit den bekannten Maßen $219 \mathrm{~mm} \times 297 \mathrm{~mm}$ vom Arbeitsplatz aus entzerrt. Die Kamera wurde senkrecht über der Arbeitsfläche platziert. Der weiße Arbeitsbereich wurde auf einer Ebene mit dunklem Hintergrund platziert, um die Erkennung von Konturen zu erleichtern. Konturen können einfach als eine Kurve erklärt werden, die alle Punkte entlang der Grenze verbindet, die die gleiche Farbe oder Intensität haben. Wir haben OpenCV-Funktionen wie cv2.canny() für die canny-Kantenerkennung und cv2.findcontours() verwendet, um alle Grenzpunkte (X, Y) eines Objekts im Bild zu finden. Das Programm berechnet diejenige Kontur mit den meisten Randpunkten, so dass die Grenzen des weißen Arbeitsbereichs aus dem Bild bekannt sind. Dann wurde mit Hilfe der Funktionen cv2.getPerspectiveTransform() und cv2.warpPerspective() Warp-Transformationen durchgeführt, um eine „Vogelperspektive“ zu erhalten. Mit anderen Worten, die perspektivische Transformation funktioniert für eine Menge von Punkten. Sie wendet die Warp-Matrix auf eine Menge von Punkten in einem Vektor an und legt die Ergebnisse in einem anderen Vektor ab. Die Warp-Perspektive kann Bild A verzerren und das Ergebnis mithilfe der Warp-Matrix in Bild B einfügen. Die Warp-Perspektive wendet dies auf die Koordinaten des Objekts an und verschiebt den Pixelwert zu einem neuen Pixel. Da wir zwei verschiedene Objekte haben, ist es hilfreich, die Abmessungen des Objekts im Bild zu messen, damit die Breite des Greifers des Roboterarms entsprechend angepasst werden kann. Um die tatsächlichen Abmessungen der Objekte zu ermitteln, haben wir Funktionen geschrieben, die den Abstand messen und entsprechend den bekannten skalieren können. Es wird festgestellt, dass die vom Programm gemessenen Abmessungen mit einem Fehler von bis $\mathrm{zu} \pm 3 \mathrm{~mm}$ ausreichend genau sind. Beispielhaft ist ein Ergebnis in der Abbildung 5 dargestellt.

Aus den Abmessungen und der Vorhersage von YOLO V4 kann die Höhe des Objekts mit dem Algorithmus, der in Bild 7 dargestellt ist, ermittelt werden. 


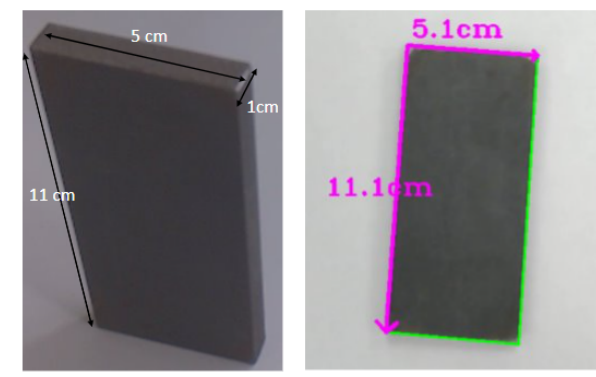

Bild 5: Tatsächliche Abmessungen des Quaders und vom Programm gemessene Abmessungen

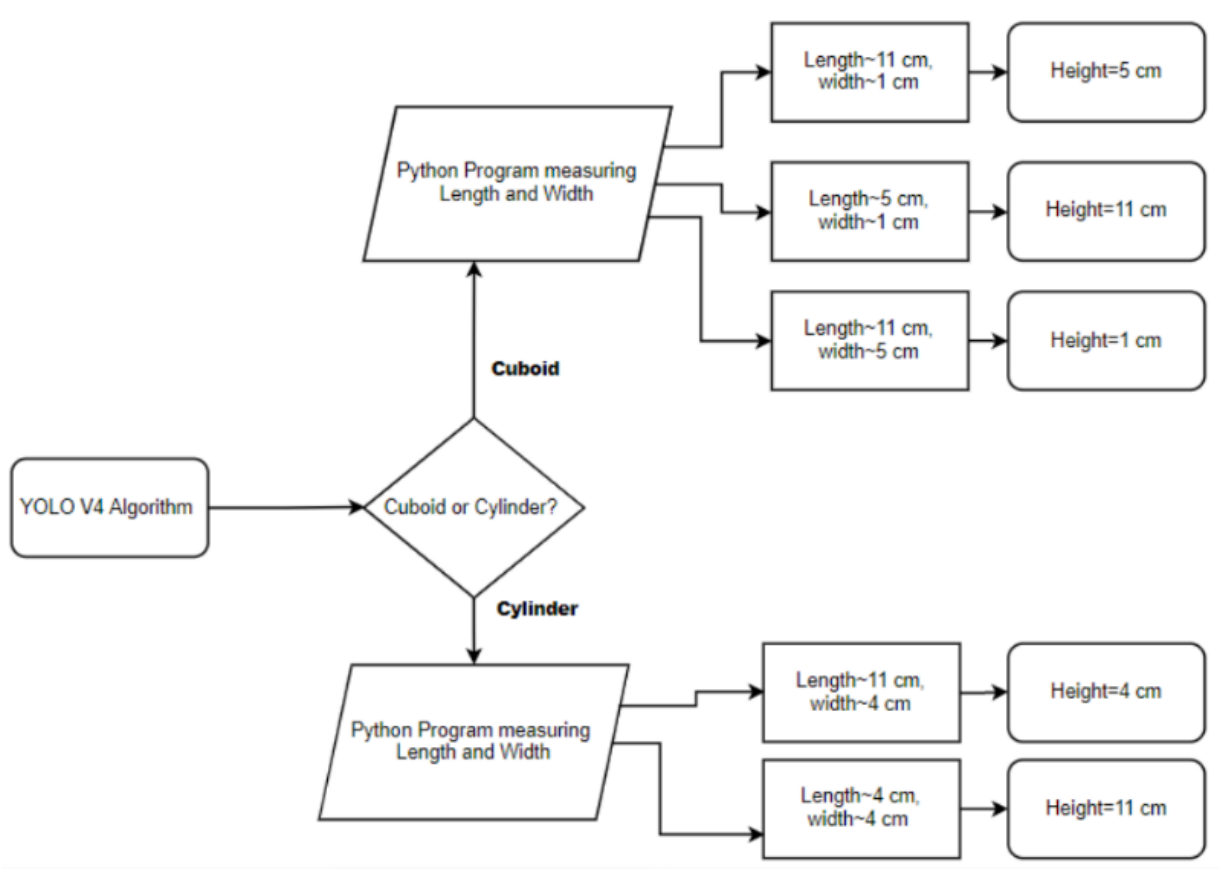

Bild 6: Beispiele von Vorhersagen des Modells zu den Objekten

Um den Schwerpunkt des Objekts zu messen, haben wir ein Programm geschrieben, das die Bounding Box um das Objekt zeichnet und die Bildmomente verwendet, um den Schwerpunkt des Objekts zu finden. Die OpenCV-Methoden cv2.boundingRect() und cv2.moments() werden zum Zeichnen des Begrenzungsrahmens und zum Ermitteln des Schwerpunkts verwendet. Bildmoment ist ein bestimmter gewichteter Durchschnitt der Bildpixelintensitäten, mit dessen Hilfe wir einige spezifische Eigenschaften eines Bildes, wie Radius, Fläche, Schwerpunkt usw., finden können.

Der gemessene Schwerpunkt hat einen Fehler von bis $\mathrm{zu} \pm 3 \mathrm{~mm}$. Es wurde beobachtet, dass die Schwerpunktmessung ungenau ist, wenn mehrere Flächen des Quaders oder Zylinders sichtbar sind. Das Bild sollte direkt über dem Objekt aufgenommen werden und nur die Oberseite des Objekts erfassen. Auch die Schwerpunktmessung für die zylindrischen Oberflächen ist aufgrund der unvollkommenen Anpassung der Begrenzungsrahmen nicht genau. Schließlich haben wir zur Messung der Drehung des Objekts auf der zweidimensionalen Ebene die OpenCV-Methode cv2.minAreaRect() verwendet, um die minimale Fläche des gedrehten Rechtecks zu ermitteln. Diese Methode nimmt als Eingabe einen 2DPunktesatz und gibt eine Bounding-Box-Struktur zurück, die die folgenden Details enthält: Mittelpunkt (X, Y), (Breite, Höhe) und Drehwinkel. 

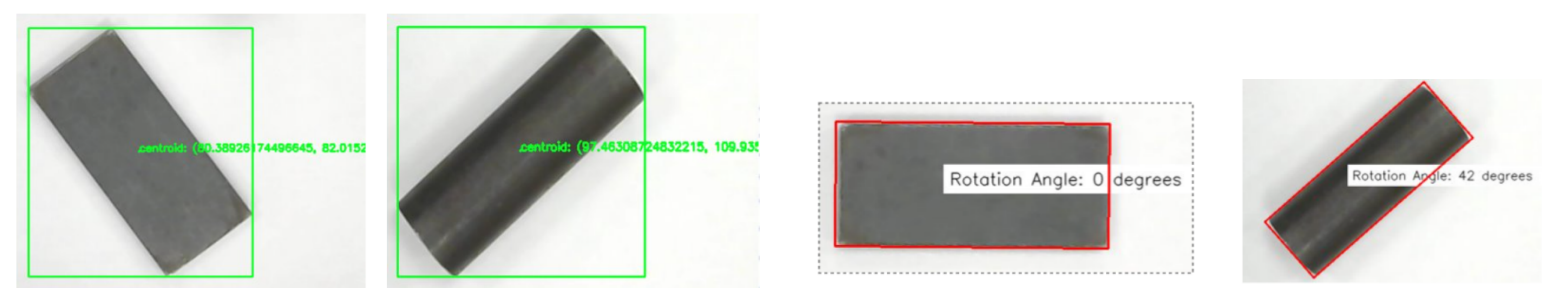

Bild 7: Die grün umrandeten Objekte zeigen vom Programm gemessene reale Koordinaten des Quaders und des Zylinders, die rot umrandeten Objekte die Drehung

\subsection{Berechnung der X-, Y- und Z-Koordinaten des Objektschwerpunkts im Be- zugssystem des Roboters}

Aus dem gemessenen Schwerpunkt können die X-, Y- und Z-Koordinaten berechnet werden, indem man einen Referenzpunkt verwendet und die Offsets, d.h. die gemessenen Schwerpunktkoordinaten, addiert. Zu diesem Zweck können dem Roboter einige Punkte manuell beigebracht werden, und die Richtungen der positiven und negativen Achsen können dabei geschätzt werden. Der Bezugspunkt ist dabei die bekannte Ecke des Arbeitsbereichs, deren Koordinaten bereits bekannt sind.

\subsection{Kommunikation zwischen Jetson Nano und Kuka KR6}

KukaVarProxy ist ein Server, der auf der KUKA Robotersteuerung läuft und mit dem internen System kommuniziert. Dieser Server hat eine TCP/IP-Schnittstelle zum Kuka-Roboter, die das Lesen und Schreiben von Variablen und Datenstrukturen der gesteuerten Manipulatoren ermöglicht. Der KukaVarProxy-Server lauscht auf dem TCP-Port 7000 auf Netzwerknachrichten, liest und schreibt Daten in die KRC-Systemvariablen. Das Pendant ist ein „HMI“, ein von KUKA bereitgestelltes Programm, das unter Windows läuft und die Schnittstelle darstellt, über die der Roboterbenutzer den Roboter bedienen kann. Um eine TCP/IP-Verbindung herzustellen, wird KukaVarProxy auf dem Betriebssystem der Steuerung ausgeführt und dann die Netzwerkverbindung von KUKA „HMI“ konfiguriert.

Ein Netzwerk-Socket ist ein Endpunkt einer prozessübergreifenden Kommunikation über ein Computernetzwerk. Die Python-Standardbibliothek verfügt über ein Modul namens socket, das eine Low-Level-Schnittstelle für die Internet-Anbindung bietet. Diese Schnittstelle steht in verschiedenen Programmiersprachen zur Verfügung, da sie Systemaufrufe auf Betriebssystemebene verwendet. Die Funktion socket akzeptiert die Argumente family, type und proto. AF_INET ist die InternetAdressfamilie für IPv4. SOCK_STREAM ist der Socket-Typ für TCP, das Protokoll, das für den Transport unserer Nachrichten im Netzwerk verwendet wird. Das Transmission Control Protocol (TCP) wird verwendet, weil es zuverlässig ist und die Daten in der richtigen Reihenfolge zustellt. Das Socket-ClientProgramm verwendet die Funktionen client.connect(), client.send(), client.recv() und client.close(), um eine Verbindung herzustellen, Daten zu senden, Informationen vom Server zu empfangen bzw. das Socket zu schließen.

Um die Verbindung herzustellen, wurde ein Objekt mit dem Namen „Kuka“ erstellt und die IP-Adresse des KukaVarProxy-Servers, der auf KRC4 läuft, gesendet.

\subsection{Programmierung der Pick-And-Place-Aufgabe des Roboters}

Es wird der Variablentyp E6POS verwendet, um die Koordinaten an den Roboter zu senden. Die E6POSVariable besteht aus 6 Variablen, die den Punkt im kartesischen Raum und die Ausrichtung des Arms an diesem Punkt darstellen. Aus diesem Grund kann der Programmierer eine E6POS-Variable auf verschiedene Weise referenzieren. Die folgenden Codezeilen wurden in die Datei config.dat geschrieben, nachdem die Benutzergruppe auf „Expertenmodus“ geändert wurde.

Zum Test wurde ein einfaches KRL-Programm definiert, bei dem der Roboter eine Punkt-zu-PunktBewegung zu dem Punkt mp[1] startet, nachdem er die Koordinaten vom PC erhalten hat. In Bild 8 ist 
das Programm auf unserem PC dargestellt, das die Koordinaten an den Roboter sendet.

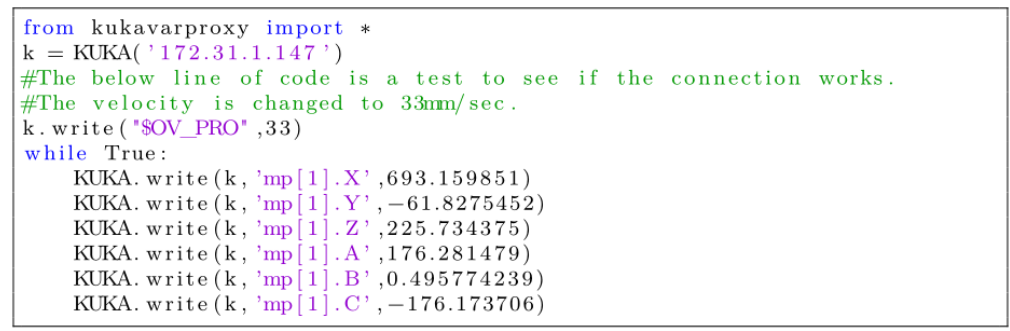

Bild 8: Python-Programm auf dem PC zum Test zum senden der Koordinaten vom PC zum Kuka

In der Abbildung 9 sind die Schritte aufgeführt, die durchgeführt werden müssen, um den autonomen Entnahme- und Ablagevorgang für den Roboter zu ermöglichen. Vor dem Greifen des Objekts wird eine LIN-Bewegung eingeführt, um eine plötzliche Kollision des Greifers mit der Oberfläche des Objekts zu vermeiden.

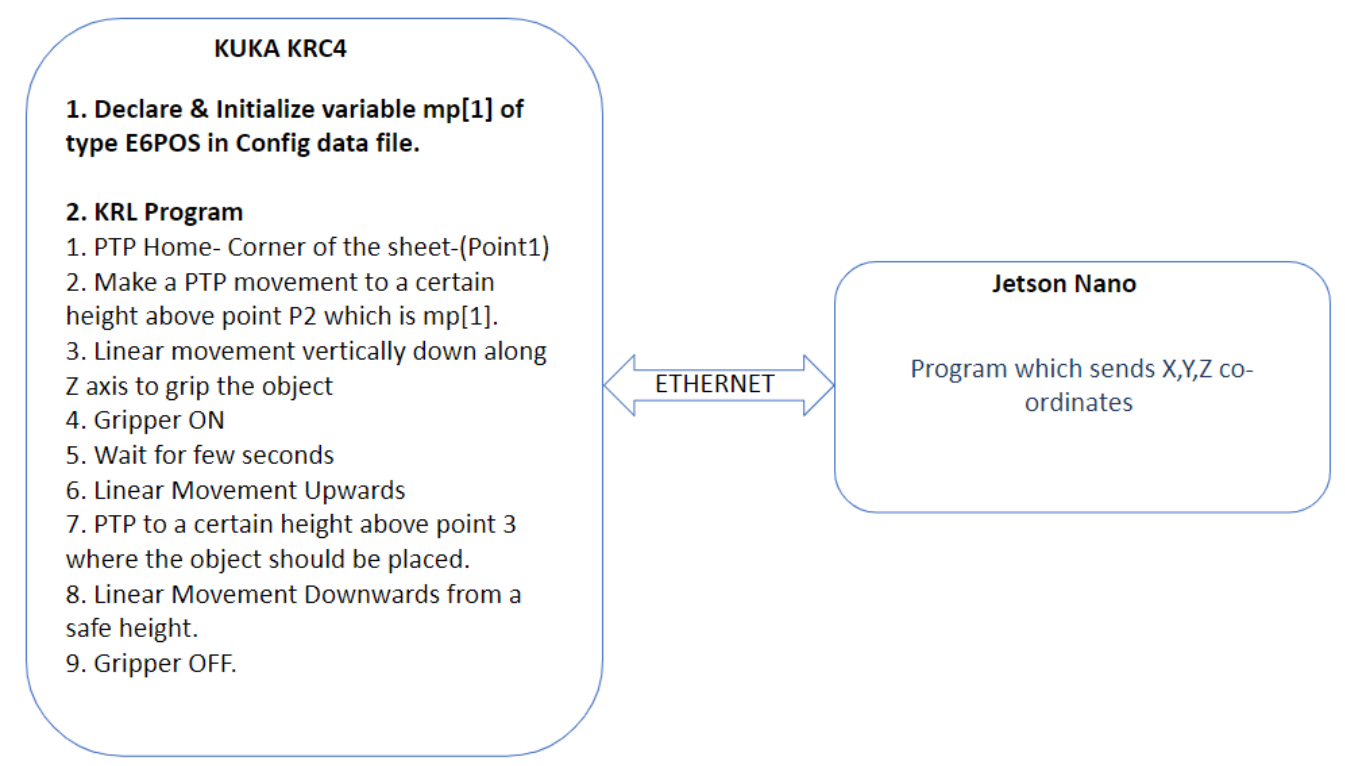

Bild 9: Ablauf des Pick-And-Place-Vorgangs

\section{Evaluierung und Ausblick}

Durch eine klare Definition der Arbeitsplatzumgebung und eine gute Beleuchtung wurde mit dem Algorithmus YOLO V4 auf dem Jetson Nano eine durchschnittliche Genauigkeit in unterschiedlichen Positionen der Objekte von $100 \%$ und eine Bildrate von 4,2 FPS erreichen. Auch der Schwerpunkt und der gemessene Drehwinkel waren bei den meisten Tests unter guten Lichtverhältnissen ausreichend genau. In einigen Fällen wurde ein Fehler von bis zu $3 \mathrm{~mm}$ aufgrund von Schattenwurf beobachtet, der mit Hilfe von Näherungsfunktionen korrigiert werden kann. Bei der Drehung des Objekts wird ein Fehler von bis zu 1 bis 2 Grad beobachtet. Die Client-Server-Architektur wurde erfolgreich eingerichtet, die Koordinaten wurden an den Roboter gesendet und die Bewegung des Roboters konnte durchgeführt werden. Die Flexibilität eines Roboters, der auf diese Weise von kostengünstigen Bildverarbeitungssystemen geführt wird, kann den Bedarf an präzise konstruierten Teilezuführungen verringern und damit die Flexibilität in der Fertigungszelle erhöhen sowie kurze Produktionsläufe und Anpassungsfähigkeit ermöglichen. 


\section{Literatur}

[1] Alexey Bochkovskiy, Chien-Yao Wang, Hong-Yuan Mark Liao: „YOLOv4: Optimal Speed and Accuracy of Object Detection“, 2020, arXiv:2004.10934

[2] P. Huang, A. K. Mok: „A Case Study of Cyber-Physical System Design: Autonomous Pick-and-Place Robot“, 2018 IEEE $24^{\text {th }}$ International Conference on Embedded and Real-Time Computing Systems and Applications (RTCSA), 2018, pp. 22-31, doi: 10.1109/RTCSA.2018.00012.

[3] Pritam Prakash Shete, Abhishek Jaju, Surojit Kumar Bose, Prabir Pal: „Stereo Vision Guided Telerobotics System for Autonomous Pick and Place Operations“, 2015. In Proceedings of the 2015 Conference on Advances In Robotics (AIR '15). Association for Computing Machinery, New York, NY, USA, Article 41, pp. 1-6. doi: $10.1145 / 2783449.2783490$

[4] T. Kotthauser, G. F. Mauer, „Vision-Based Autonomous Robot Control for Pick and Place Operations“, IEEE/ASME International Conference on Advanced Intelligent Mechatronics, 2009, pp. 1851-1855, doi: 10.1109/AIM.2009.5229792.

[5] P. Andhare, S. Rawat, „Pick and Place Industrial Robot Controller with Computer Vision“. International Conference on Computing Communication Control and automation (ICCUBEA), 2016, pp. 1-4, doi: 10.1109/ICCUBEA.2016.7860048.

[6] U. Fayyad, G. Piatetsky-Shapiro, P. Smyth: „The KDD Process for Extracting Useful Knowledge from Volumes of Data“. 1996, Commun. ACM, 39, pp. 27-34.

[7] C. Cattaneo, G. Mainetti, R. Sala: „The Importance of Camera Calibration and Distortion Correction to Obtain Measurements with Video Surveillance Systems“. 2015, Journal of Physics: Conference Series, doi: 658. 012009. 10.1088/1742-6596/658/1/012009.

[8] Yu S., Zhu R., Yu L., Ai W.: „Effect of Checkerboard on the Accuracy of Camera Calibration“. 2018, doi:10.1007/978-3-030-00764-5_57. 